\title{
Quench, thermalization, and residual entropy across a non-Fermi liquid to Fermi liquid transition
}

\author{
Arijit Haldar, ${ }^{1,2, *}$ Prosenjit Haldar,,${ }^{1,3}$ Surajit Bera, ${ }^{1}$ Ipsita Mandal, ${ }^{4,5}$ and Sumilan Banerjee ${ }^{1, \dagger}$ \\ ${ }^{1}$ Centre for Condensed Matter Theory, Department of Physics, Indian Institute of Science, Bangalore 560012, India \\ ${ }^{2}$ Department of Physics, University of Toronto, 60 St. George Street, Toronto, Ontario, M5S 1A7, Canada \\ ${ }^{3}$ Laboratoire de Physique Théorique, IRSAMC, Université de Toulouse, CNRS, UPS, France \\ ${ }^{4}$ Laboratory of Atomic and Solid State Physics, Cornell University, Ithaca, New York 14853, USA \\ ${ }^{5}$ Faculty of Science and Technology, University of Stavanger, 4036 Stavanger, Norway
}

(Received 12 April 2019; revised manuscript received 12 February 2020; accepted 13 February 2020; published 12 March 2020)

\begin{abstract}
We study the thermalization, after sudden and slow quenches, in an interacting model having a quantum phase transition from a Sachdev-Ye-Kitaev (SYK) non-Fermi liquid (NFL) to a Fermi liquid (FL). The model has SYK fermions coupled to noninteracting lead fermions and can be realized in a graphene flake connected to external leads. A sudden quench to the NFL leads to rapid thermalization via collapse-revival oscillations of the quasiparticle residue of the lead fermions. In contrast, the quench to the FL shows multiple prethermal regimes and much slower thermalization. In the slow quench performed over a time $\tau$, we find that the excitation energy generated has a remarkable intermediate- $\tau$ nonanalytic power-law dependence, $\tau^{-\eta}$ with $\eta<1$, which seemingly masks the dynamical manifestation of the initial residual entropy of the SYK fermions. Our study gives an explicit demonstration of the intriguing contrasts between the out-of-equilibrium dynamics of a NFL and a FL in terms of their thermalization and approach to adiabaticity.
\end{abstract}

DOI: 10.1103/PhysRevResearch.2.013307

\section{INTRODUCTION}

One of the major frontiers in condensed-matter physics is to describe gapless phases of interacting fermions without any quasiparticles, namely non-Fermi liquids (NFL) [1]. Recently, new insights about fundamental differences between NFLs and Fermi liquids (FL) have been gained in terms of manybody quantum chaos and thermalization. This new impetus has come from exciting developments in a class of NFLs described by the Sachdev-Ye-Kitaev (SYK) model [2-4] and its extensions [5-13] and their connections with black holes in quantum gravity $[3,14,15]$. In particular, the model proposed in Ref. [6] classifies the SYK NFL and a FL as two distinct chaotic fixed points separated by a quantum phase transition (QPT). In this characterization, the NFL thermalizes much faster than the FL, as quantified by a rate of the onset of chaos or the Lyapunov exponent $[3,6,16]$.

However, the Lyapunov exponent is computed from an equilibrium dynamical correlation, the so-called out-of-timeordered correlator $[3,4,17]$. Here, using the model of Ref. [6] as a template, we ask whether such contrast between the NFL and FL persists even for thermalization from a completely outof-equilibrium situation, e.g., a quantum quench. The model

\footnotetext{
*arijithaldar@iisc.ac.in

†sumilan@iisc.ac.in
}

Published by the American Physical Society under the terms of the Creative Commons Attribution 4.0 International license. Further distribution of this work must maintain attribution to the author(s) and the published article's title, journal citation, and DOI. has two species of fermions, interacting SYK fermions coupled to another species of otherwise noninteracting fermions, referred to as lead fermions. A QPT between a strongly interacting NFL and weakly interacting FL phases can be tuned in the model at low energies by varying the ratio, $p$, of numbers of sites on which the two types of fermions reside. Remarkably, the solvable nature of the model allows us to study its full nonequilibrium evolution after a quench exactly. By using nonequilibrium Keldysh field theory in the thermodynamic limit, as well as numerical exact diagonalization (ED) for finite systems, we demonstrate a drastic difference in thermalization rates for the NFL and FL after a sudden quench. In addition, we show that the quasiparticle residue of the lead fermions exhibits a dynamical transition as function of $p$ from collapse-and-revival oscillations to prethermalized plateaus as a function of time. This dynamical transition is similar to that seen in the interaction quench of Hubbard model [18].

Furthermore, the Landau description of a FL is based on the concept of adiabatic time evolution from a noninteracting system under slow switching on of the interaction, without encountering a phase transition. Is it possible to evolve an NFL adiabatically to the FL and vice versa? We argue that such evolution is not possible here due to another intriguing aspect of the SYK NFL, namely the finite zero-temperature residual entropy (density) $S_{0}[3,4,15]$. The entropy is related to the Bekenstein-Hawking entropy of the black hole in the dual gravity theory $[3,4,15,17]$ and has relevance for strange metallic states described by local quantum criticality $[5,9,11,13]$. We probe the signature of this entropy in the heat generated during nonequilibrium dynamics and characterize how the putative adiabatic limit is approached in the two phases, and across the QPT, after a slow quench with a finite rate. We show 
that, remarkably, the heat or energy of excitations $\Delta E$ generated by the quench scales as $\Delta E(\tau) \sim \tau^{-\eta}$ with quench time $\tau$. Moreover, we find a direct manifestation of the equilibrium QPT in the scaling exponent $\eta$. We also contrast all the above results for the interacting model with those for an analogous noninteracting model under identical quench protocols. In particular, we show that the two models show drastically different thermalization behaviors in the thermodynamic limit.

The model studied here could be realized in a graphene nano flake [19] attached to leads and under a magnetic field. The QPT also has close parallel in the NFL-to-FL transition in the multichannel Kondo model [20]. Moreover, the study of dynamics after a quench in our model, where no quasiparticle description exists in one of the phases around the QPT, allows us to probe hitherto unexplored regime of many-body quantum dynamics. This is complementary to the previous studies of dynamics after quench across a QPT in integrable models $[21,22]$ or weakly interacting systems with well-defined quasiparticles $[18,23,24]$. The scaling laws mentioned above cannot be explained by the usual KibbleZurek scaling $[25,26]$, unlike that in integrable or weakly integrable models $[18,21-24]$. Although there have been a few studies on nonequilibrium dynamics of the original SYK model [27-30], none of them addressed the issue of a quench across a nontrivial QPT.

The remainder of this paper is organized as follows. In Sec. II, we introduce the interacting and noninteracting models and describe the quench protocols. Section III discusses the results for the nonequilibrium evolutions after slow and sudden quenches in the large- $N$ limit obtained using nonequilibrium Schwinger-Keldysh method. Some results for finite- $N$ obtained via ED studies in a few limiting cases are also discussed in this section. In Sec. IV we conclude with the implications and significance of our results. The details of the nonequilibrium large- $N$ formulations, equilibrium spectral properties of the models, and some additional results on the slow quench in the noninteracting model are included in Appendices A-C. The analysis of the slow quench using usual adiabatic perturbation theory and the breakdown of adiabaticity in our model are discussed in Appendix D. Additional details of the numerical calculations, ED, and the results are given in the Supplemental Material [31].

\section{MODEL}

\section{A. Interacting model}

As described schematically in Fig. 1, we study a time( $t$ ) dependent version of the model in Ref. [6], $\mathcal{H}(t)=\mathcal{H}_{c}+$ $\mathcal{H}_{\psi}+\mathcal{H}_{c \psi}(t)$, where

$$
\begin{aligned}
\mathcal{H}_{c} & =\frac{1}{(2 N)^{3 / 2}} \sum_{i j k l} J_{i j k l} c_{i}^{\dagger} c_{j}^{\dagger} c_{k} c_{l}, \\
\mathcal{H}_{\psi} & =\frac{1}{M^{1 / 2}} \sum_{\alpha \beta} t_{\alpha \beta}^{\psi} \psi_{\alpha}^{\dagger} \psi_{\beta}, \\
\mathcal{H}_{c \psi}(t) & =\frac{f(t)}{(N M)^{1 / 4}} \sum_{i \alpha}\left(V_{i \alpha} c_{i}^{\dagger} \psi_{\alpha}+V_{i \alpha}^{*} \psi_{\alpha}^{\dagger} c_{i}\right) .
\end{aligned}
$$

The model (Fig. 1), has two species of fermions: (1) the SYK fermions $(c)$, on sites $i=1, \ldots, N$, interacting with random four-fermion coupling $J_{i j k l}$ [Eq. (1a)], drawn from a

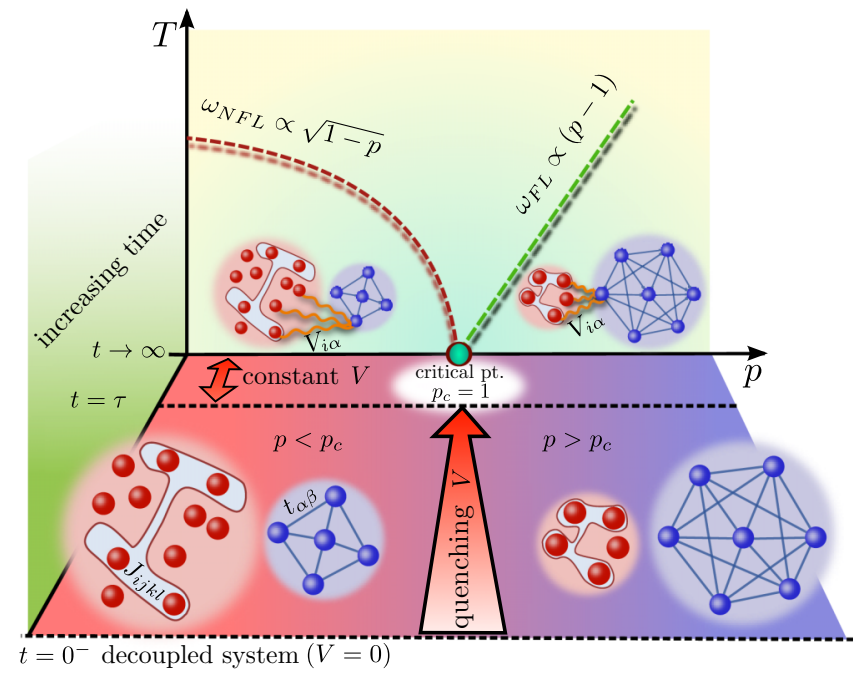

FIG. 1. Model and the quench protocol: SYK $(c)$ fermions (red dots), interacting via random quartic coupling $J_{i j k l}$ on $N$ sites, are connected, at time $t=0$, to lead $(\psi)$ fermions (blue dots joined by lines) using random-quadratic couplings $V_{i \alpha}$ with strength $V$. The $\psi$ fermions reside on $M$ sites and have random hopping amplitudes $t_{\alpha \beta}^{\psi}$. For a fixed site fraction $p=M / N$, the coupling $V$ is ramped from 0 to a finite value over quench duration $\tau$, as depicted by a red arrow pointing into the page. For $t \rightarrow \infty$, the connected system is expected to relax to a thermal state in the equilibrium $p-T$ phase diagram, where $T$ is the final temperature. A critical point, $p_{c}=1$, separates the SYK NFL $\left(p<p_{c}\right)$ and the FL $\left(p>p_{c}\right)$. The low-energy NFL and FL behaviors persist up to the crossover scales $\omega_{\mathrm{NFL}}$ and $\omega_{\mathrm{FL}}$, respectively.

Gaussian distribution with zero mean and variance $\overline{\left|J_{i j k l}\right|^{2}}=$ $J^{2}$, and (2) the lead fermions $(\psi)$, on a separate set of sites $\alpha=1, \ldots, M$ connected via random all-to-all hopping $t_{\alpha \beta}^{\psi}$ [Eq. (1b)]. The SYK and the lead fermions are quadratically coupled via $V_{i \alpha} ; t_{\alpha \beta}^{\psi}$ and $V_{i \alpha}$ are complex Gaussian random variables with zero mean and variances $\overline{\left|t_{\alpha \beta}^{\psi}\right|^{2}}=t_{\psi}^{2}$ and $\overline{\left|V_{i \alpha}\right|^{2}}=V^{2}$, respectively.

The model is exactly solvable for $N, M \rightarrow \infty$ with a fixed ratio $p=M / N$ that is varied to go through the QPT between NFL and FL at a critical value $p=p_{c}=1$ [6]. Two crossover scales, $\omega_{\mathrm{NFL}}$ and $\omega_{\mathrm{FL}}$, approach zero from either side of the QPT (Fig. 1). The residual entropy density $S_{0}(p)$ of the SYK NFL continuously vanishes at the transition [6]. This is one of the unique features of the QPT.

To probe the nonequilibrium dynamics, we make the coupling term in Eq. (1c) time dependent. In particular, we perform geometric quenches (Fig. 1) by switching on the coupling between the two initially disconnected subsystems (a) suddenly such that $f(t)=\Theta(t)$, the Heaviside step function, and (b) by slowly ramping up the coupling over a time $\tau$, i.e., $f(t)=r(t / \tau)[\Theta(t)-\Theta(t-\tau)] ; r(x)$ is a ramp function, e.g., $r(x)=x$. Before the quench, the disconnected subsystems, with a preset site ratio $p$, are at their own thermal equilibria at initial temperatures $T_{i}^{c}$ and $T_{i}^{\psi}$. We take $T_{i}^{c}, T_{i}^{\psi} \rightarrow 0$ so that SYK and lead fermions belong to the NFL and (noninteracting) FL states, respectively. As shown in Fig. 1 , for $t \rightarrow \infty$, depending on whether $p<1$ or $p>1$, the coupled system 
eventually is expected to thermalize to either the NFL or the FL state, respectively. In any case, one of the subsystems always undergoes a transition, either from FL to NFL or vice versa, under the quench.

\section{B. Noninteracting model}

To contrast the behavior of the above interacting model, and to demonstrate the crucial role of interaction in the nonequilibrium dynamics after quench and eventual thermalization, we also consider an analogous noninteracting model. The latter is obtained by replacing the interaction term for the $c$ fermions with a random hopping term similar to the one appearing in the $\psi$-fermion Hamiltonian [Eq. (1)]. To this end, we have $\mathcal{H}^{(\mathrm{NI})}(t)=\mathcal{H}_{c}+\mathcal{H}_{\psi}+\mathcal{H}_{c \psi}(t)$, with

$$
\mathcal{H}_{c}^{(\mathrm{NI})}=\frac{1}{(N)^{1 / 2}} \sum_{i j} t_{i j}^{c} c_{i}^{\dagger} c_{j} .
$$

Here $t_{i j}^{c}$ is a complex Gaussian random variable with zero mean and variance $\overline{\left|t_{i j}^{c}\right|^{2}}=t_{c}^{2} ; \mathcal{H}_{\psi}$ and $\mathcal{H}_{c \psi}(t)$ are the same as in Eq. (1).

Below, we first briefly discuss the method for studying the time evolution of the systems, followed by the results for sudden and slow quenches.

\section{RESULTS}

\section{A. Nonequilibrium evolution}

We use standard Schwinger-Keldysh nonequilibrium Green's function technique [32,33] to study the quenches described above. Utilizing the closed-time-contour Schwinger-Keldysh action (see Appendix A) for the model, we derive the Kadanoff-Baym (KB) equations for the disorder-averaged nonequilibrium Green's functions, $G_{s}^{<}\left(t_{1}, t_{2}\right), \quad G_{c}^{>}\left(t_{1}, t_{2}\right) \quad(s=c, \psi), \quad$ e.g., $\quad G_{c}^{>}\left(t_{1}, t_{2}\right)=$ $-i\left\langle c_{i}\left(t_{1}\right) c_{i}^{\dagger}\left(t_{2}\right)\right\rangle$; the overline denotes disorder averaging. The KB equations are numerically integrated using a predicator-corrector scheme (see the Supplemental Material [31], Sec. 1) starting from the initial equilibrium Green's functions (see the Supplemental Material [31], Sec. 2) for the disconnected system. The time dependence of $\mathcal{H}(t)$ is encoded in $\mathrm{KB}$ equations via the local self-energies $\Sigma_{s}$, which could be exactly calculated in the large- $N$ limit for both the interacting and the noninteracting models (see Appendix A).

We obtain the time-dependent expectation value of an observable $\mathcal{O}(t)$, i.e., $\langle\mathcal{O}(t)\rangle \equiv \operatorname{Tr}[\rho(t) \mathcal{O}(t)]$ (see the Supplemental Material [31], Sec. 3), using the Green's functions. Here $\rho(t)$ is the time-dependent density matrix and $\mathcal{O}(t)$ includes the explicit time dependence, if any, of the observables. To understand thermalization, we track how the contributions of the individual terms in Eq. (1) to the total energy $E(t)=\langle\mathcal{H}(t)\rangle$, e.g., $E_{c \psi}(t)=\left\langle\mathcal{H}_{c \psi}(t)\right\rangle$, relax after the quench. Since the whole system is isolated, we estimate the expected temperature $T_{f}$ of the putative thermal state at long times from the total energy $E(t)=E_{f}$, which is conserved after the quench.

\section{B. Sudden quench}

We first ask whether the contrast between dynamics of the NFL and FL can be seen even when the system is subjected to an abrupt nonequilibrium process. To address this, we study the case when the subsystems are suddenly connected at $t=$ 0 . We take $J=1, t_{\psi}=1, V=1$ and low initial temperatures, $T_{i}^{c}=0.05$ and $T_{i}^{\psi}=0$ [34]. The sudden quench leads to a rather high final temperature $T_{f} \sim 1$ (see the Supplemental Material [31], Sec. 4.1). Before the quench, the lead fermions are noninteracting and the single-fermionic excitations are sharply defined at $T_{i}^{\psi}=0$. To track the quasiparticle evolution, we compute an energy-resolved time-dependent occupation, $n_{\psi}(\epsilon, t)=-i G_{\psi}^{<}(\epsilon ; t, t)$, for the lead fermions. Here $\epsilon$ are the eigenvalues of the quadratic Hamiltonian in Eq. (1b), i.e., $\mathcal{H}_{\psi}=\sum_{\epsilon} \epsilon \psi_{\epsilon}^{\dagger} \psi_{\epsilon}$, and the Green's function $G_{\psi}^{<}\left(\epsilon ; t_{1}, t_{2}\right)$ is obtained by integrating an appropriate $\mathrm{KB}$ equation [see Eq. (A6)].

The quasiparticle residue, $z_{\psi}(t)=n_{\psi}\left(0^{-}, t\right)-n_{\psi}\left(0^{+}, t\right)$, is obtained from the occupation discontinuity at $\epsilon=0$. The vanishing of the residue indicates the destruction of the quasiparticles.

\section{Collapse-and-revival oscillations and prethermal plateaus}

In our model, $z_{\psi}^{\infty}=z_{\psi}(t \rightarrow \infty)$ is expected to vanish for quench to any $p$ since the coupled system either thermalize to NFL or to a finite-temperature FL state. As shown in Figs. 2(a) and 2(b), the collapse of the residue happens through two very different routes. First, for $p<p_{c}^{\text {dyn }}$, a critical value of $p$ corresponding to a dynamical transition, $z_{\psi}(t)$ undergoes collapse-and-revival oscillations [Fig. 2(a)]. Second, for $p>p_{c}^{\mathrm{dyn}}, z_{\psi}(t)$ shows multiple long prethermal plateaus [Fig. 2(b)]. For $V=1$, we find $p_{c}^{\text {dyn }} \approx 1.5$ from $z_{1}(p) \rightarrow 0$, where $z_{1}$ is the residue at the first maximum of the oscillations [see curve labeled "int." in Fig. 2(a), inset]. Hence, the critical value $p_{c}^{\text {dyn }}$ for this dynamical transition is greater than the "equilibrium" critical ratio $p_{c}=1$. It is encouraging to find that similar oscillations and evidence of a dynamical transition have been observed [18] in the interaction quench across Mott transition in the Hubbard model as well.

However, in contrast to the interaction quench in the Hubbard model, where the collapse-and-revival oscillations originate from on-site Hubbard repulsion, in our model, the oscillations are linked to a soft hybridization gap in the lead fermions. The gap appears due to hybridization [Eq. (1c)] between the SYK and lead fermions and closes at the NFL-FL transition (Appendix B). In fact, we observe a similar dynamical transition in the noninteracting model of Eq. (2) under an analogous sudden quench [see the curve labeled "n.int." in Fig. 2(a), inset]. The critical point in this scenario occurs at $p_{c}^{\text {dyn }} \approx 0.5$. We emphasize here that although a dynamical transition exists for the noninteracting case, the presence of interactions is crucial for thermalization (discussed in the next section), which the noninteracting model fails to achieve for any value of $p$ (see the Supplemental Material [31], Sec. 4.3). The SYK interactions are also responsible for nontrivially shifting the critical value of $p_{c}^{\text {dyn }}$ from 0.5 to 1.5 as shown in Fig. 2(a) (inset).

\section{Thermalization and long-time steady states}

The crucial aspects that distinguish the noninteracting [Eq. (2)] and interacting [Eq. (1)] models, as well as the 


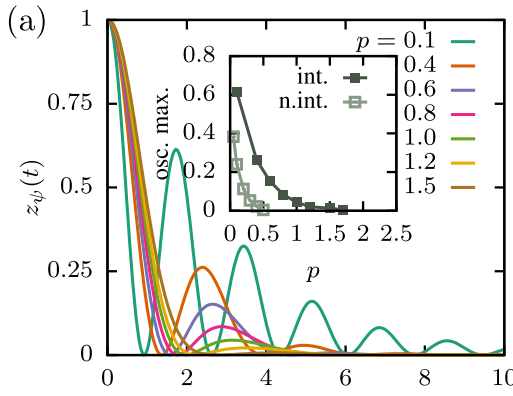

(e)

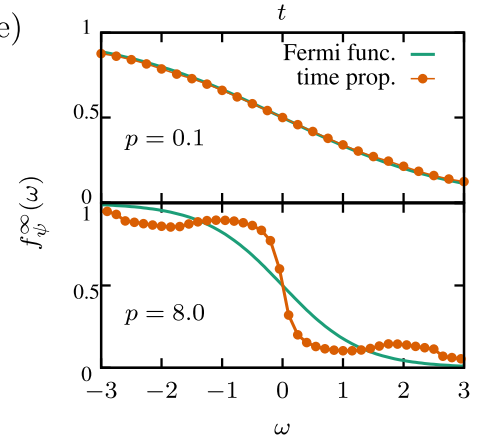

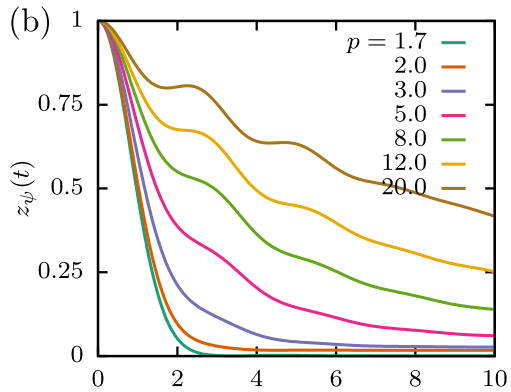

(f)

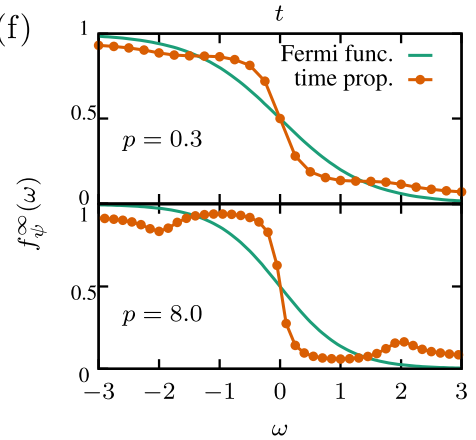

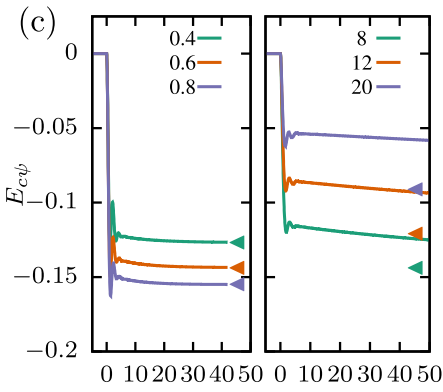

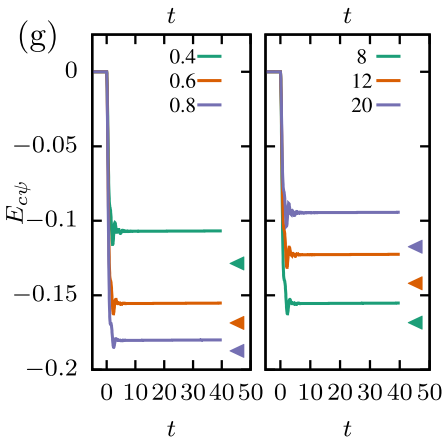

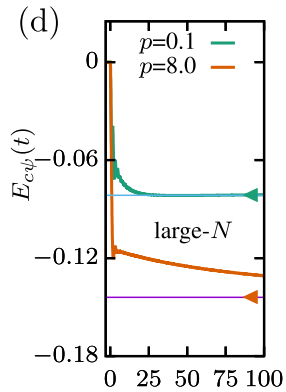

$(\mathrm{h})$

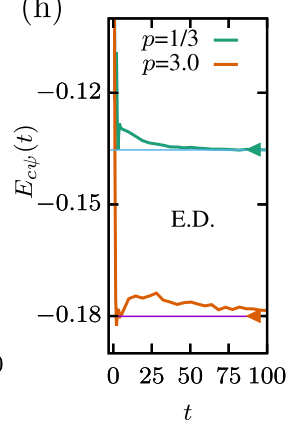

FIG. 2. Dynamical transition and thermalization after a sudden quench: Panels (a) and (b) show the quasiparticle residue $z_{\psi}(t)$ for the ranges $p=0.1-1.5$ and $p=1.7-20.0$, respectively. In (a) inset, the curve labeled "int." shows the height $z_{1}$ of the first maximum of the oscillations as a function of $p$. This is compared with the $p$ dependence of the oscillation maximum (marked as "n.int."), when the $c$ fermions are made noninteracting. The effect of interactions pushes the critical value of $p_{c}^{\text {dyn }}$ from 0.5 to 1.5 . Panel (c) gives the time evolution of bond energy $E_{c \psi}$, in the interacting model, for $p=0.4-0.8$ (left) and $p=8-20$ (right), while panel (g) shows the time evolution in the noninteracting model for the same $p$ values. The $E_{c \psi}$ attain their respective equilibrium values (triangles) rather quickly when $p<p_{c}^{\text {dyn }}$ in the interacting model and shows a slow approach to thermalization when $p \gg p_{c}^{\text {dyn }}$. However, thermalization is completely absent in the noninteracting model, and $E_{c \psi}$ never reach their respective equilibrium values regardless of the value of $p$. Panel (e) shows the $\psi$-fermion long-time steady-state occupation function $f_{\psi}^{\infty}(\omega)$ (dots) in the interacting model for $p=0.1$ (top) and 8.0 (bottom), respectively, and compares them with the Fermi function $n_{F}\left(\omega, T_{f}\right)$ (line). Thermalization occurs for $p=0.1$, i.e., $f_{\psi}^{\infty}(\omega) \rightarrow n_{F}\left(\omega, T_{f}\right)$, while $p=8.0$ remains athermal. Panel (f) shows the failure of $f_{\psi}^{\infty}(\omega)$ (dots) for the noninteracting model to approach $n_{F}\left(\omega, T_{f}\right)$ (line) for both $p=0.3,8.0$. Panels (d) and (h) give $E_{c \psi}$ as a function of $t$ obtained via large- $N$ calculation for $p=0.1,8.0$ and via ED for $p=1 / 3,3.0$, respectively, for the interacting model. The thermal expectation (diagonal ensemble) values for the large- $N$ (ED) case are shown by the arrow heads and horizontal lines.

NFL and FL, are the thermalization process and the long-time steady states. As shown in Fig. 2(c) [also see the Supplemental Material [31], Sec. 4.2, Figs. S3(a) and S3(b)], $E_{c \psi}(t)$ reaches the thermal expectation corresponding to the temperature $T_{f}$ very rapidly for $p<p_{c}^{\text {dyn }}$, whereas there is a drastically slow, albeit finite, relaxation rate for $E_{c \psi}(t)$ toward the thermal value for $p>p_{c}^{\text {dyn }}$. In contrast, $E_{c \psi}(t)$ does not relax to the expected thermal value for any $p$ in the noninteracting model, see Fig. 2(g) [also see the Supplemental Material [31], Sec. 4.2, Figs. S4(a) and S4(b)]. We further analyze the steady state through the Green's functions $G_{s}(\mathcal{T}, \omega)=\int_{-\infty}^{\infty} G_{s}\left(t_{1}=\mathcal{T}+\right.$ $\left.t / 2, t_{2}=\mathcal{T}-t / 2\right) e^{i \omega t}$, where $\mathcal{T}=\left(t_{1}+t_{2}\right) / 2$. In the steady state, $G_{s}(\mathcal{T}, \omega)$ becomes independent of $\mathcal{T}$. Moreover, for a thermal steady state at $T_{f}$, the steady-state occupation function $f_{s}^{\infty}(\omega)=\lim _{\mathcal{T} \rightarrow \infty} \mathfrak{i} G_{s}^{<}(\mathcal{T}, \omega) /\left[2 \operatorname{Im} G_{s}^{R}(\mathcal{T}, \omega)\right]$ should be equal to the Fermi function $n_{F}\left(\omega, T_{f}\right)=1 /\left(e^{\omega / T_{f}}+1\right)$, i.e., should satisfy the fluctuation-dissipation theorem (FDT). We find that for the interacting model [Eq. (1)], $f_{s}^{\infty}(\omega)=$ $n_{F}\left(\omega, T_{f}\right)$ [see Fig. 2(e), top] for the NFL $\left(p<p_{c}^{\text {dyn }}\right)$, whereas FDT gets violated [Fig. 2(e), bottom] in the FL regime $(p>$ $\left.p_{c}^{\text {dyn }}\right)$ for the largest $\mathcal{T}\left(\sim 100 J^{-1}\right.$, where $J$ is the interaction strength in Eq. (1a)) accessed.

We find that the FDT is never satisfied for the noninteracting model at any $p$, as seen in Fig. 2(f) [also see the Supplemental Materal [31], Sec. 4.2, Figs. S4(c) and $\mathrm{S} 4(\mathrm{~d})]$. This is expected for the noninteracting case, where the long-time steady state is described by a generalized Gibbs ensemble (GGE) instead of the usual thermal Gibbs ensemble [21,22,26,31]. Nevertheless, even in the interacting model, the FL phase shows an approximate GGE behavior by attaining a prethermal steady state within the time accessible in our numerical calculations. The prethermal GGE will presumably relax to a thermal state over a much longer timescale [22,35]. Similar behaviors have been seen for quenches to FL phases in other interacting models [36]. In contrast, the strong interaction leads to rapid thermalization for the NFL phase. Hence the sudden quench in the interacting model demonstrates drastically different thermalization dynamics between the NFL and the FL phases.

It is worthwhile to ask whether the contrast in the thermalization behaviors of NFL and FL persists even at finite $N$. In Fig. 2(h), we show (see also the Supplemental Material [31], Sec. 4.2) the results for $E_{c \psi}(t)$ obtained from ED studies of the model of Eq. (1) for $N=16$. The ED gives results similar to that at large $N$, shown in Fig. 2(g). Another pertinent question is whether the thermalization times of $E_{c \psi}(t)$ in NFL and FL phase can be directly related to their respective Lyapunov timescales $\left(\tau_{L}\right)[6]$. Here it is important to note that the sudden 

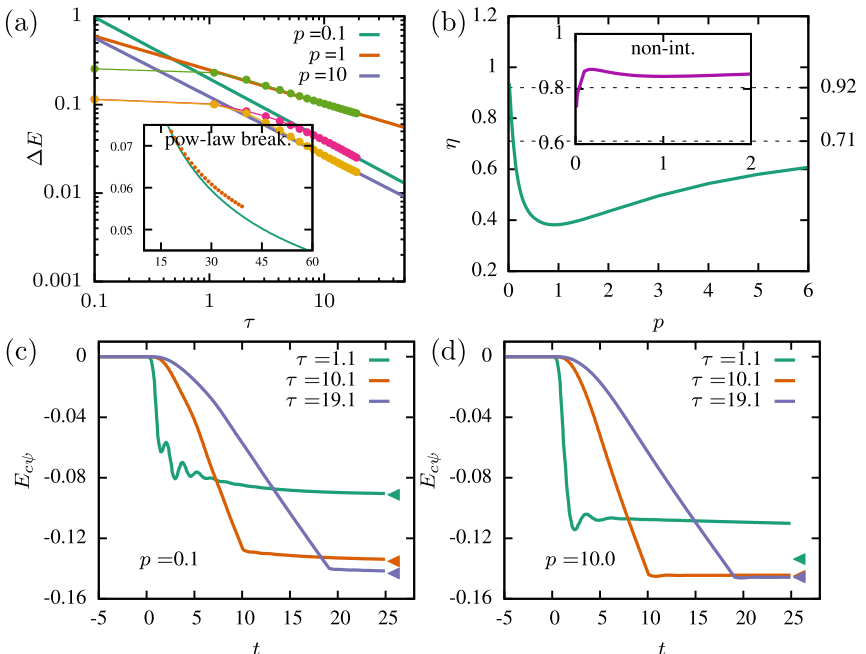

FIG. 3. Heat production and thermalization in a slow quench: (a) Heat or excitation energy $\Delta E(\tau)$ produced during the quench as a function of quench duration $\tau$ (dots) and the power-law scaling fits, $\Delta E \sim \tau^{-\eta}$ (lines), for $p=0.1,1,10$. Inset shows an indication of the deviation from the power law for larger $\tau$ at $p=10$. (b) The exponent $\eta$ vs. $p$. The inset shows the $\eta(p)$ obtained for the quench in the noninteracting model, see Eq. (2). Panels (c) and (d) show time evolutions of $E_{c \psi}$ for $\tau=1.1,10.1,19.1$, at $p=0.1$ and $p=10$, respectively. The thermal expectations are shown by the arrow heads.

quench in our model leads to (see the Supplemental Material [31], Sec. 4.1, Fig. S2) substantially high temperature $T_{f} \gtrsim$ $J$. As a result, the relaxation of various high-energy modes also influence $E_{c \psi}(t)$, making it hard to isolate $\tau_{L}$ from the relaxation of high-energy modes.

We also note that even though some specific low-energy features of SYK NFL and FL phases, like the temperature dependence of the Lyapunov time $\tau_{L}$, cannot be ascertained from the dynamics after sudden quench, our results show that the low-energy fixed points for the initial states still drastically influence the thermalization process. This is despite the fact that the sudden quench leads to substantially high final temperatures.

\section{E. Slow quench}

We next address the question whether the initial decoupled NFL and FL subsystems can be adibatically evolved to the final states of the coupled system. To this end, we consider the slow quench where the coupling is changed slowly through a ramp, $r(t / \tau)$. Here we keep both the subsystems at some low initial temperature $T_{i}^{c}=T_{i}^{\psi}=T_{i}$ for $t<0$ and define the heat or excitation energy [37,38], $\Delta E(\tau)=E(\tau)-\langle\mathcal{H}(\tau)\rangle_{T_{i}}$, produced during the quench; $\langle\mathcal{H}(\tau)\rangle_{T_{i}}$ is the thermal expectation of the final Hamiltonian $\mathcal{H}(\tau)$ at the initial temperature $T_{i}$. As shown in Fig. 3(a), remarkably, we find that $\Delta E(\tau) \sim$ $\tau^{-\eta(p)}$ with $\eta(p)<1$, i.e., a nonanalytic power-law scaling. The exponent $\eta$ has a strong nonmonotonic dependence on $p$ with a minimum around the QPT [Fig. 3(b)], revealing signatures of equilibrium QPT in the nonequilibrium evolution. We also find nonanalytic scaling for the quench in the noninteracting model (see Appendix C). However, the exponent has a very weak dependence on $p$ [Fig. 3(b), inset]. The particular nonanalytic power laws cannot be explained through a standard adiabatic perturbation theory [25,38-40], as we show in Appendix D. Also, a Kibble-Zurek-type argument $[25,26]$ cannot be given for such a zero-dimensional system. We find the exponent to depend on ramp shape as well (see Supplemental Material [31], Sec 5.1). This is also not expected from adiabatic perturbation theory for an exponent $\eta<1[31,38]$.

One possible promising route to understand the nonstandard exponent $\eta(p)$ in the intermediate time window after the quench could be to construct a low-energy theory for the model of Eq. (1) along the line of Schwarzian theory for the pure SYK model [16,17]. A recent study [41] analyzes the quench dynamics using the Schwarzian theory for an SYK model suddenly coupled to a large thermal bath made out of another SYK model. However, the situation is somewhat more involved in our model due the strong back action of SYK fermions on the lead fermions in the NFL phase $(p<1)$ and that of the lead fermions on the SYK fermions in the FL phase $(p>1)$. Such back action is absent in the model of Ref. [41] since the bath is infinitely larger than the system and since both the bath and the system are described by SYK model. In our case, one needs to start with two uncoupled low-energy theories, one corresponding to the Schwarzian action for the SYK fermions with scaling dimension $\Delta=1 / 4$, and the other for the noninteracting fermions with scaling dimension $\Delta=$ $1 / 2$. Hence the resulting theory after the quench will not be that of the standard Schwarzian mode but a different and more complicated one that includes the strong back actions among the subsystems. We would discuss this effective theory elsewhere [42].

As alluded to earlier, for the quench to any finite $p$, e.g., from the NFL to FL, the residual entropy $S_{0}$ of the NFL implies a violation of adiabaticity even for an arbitrary slow quench. The excitation energy $\Delta E(\tau)$ characterizes how $S_{0}$ metamorphoses into thermal excitations in the FL. The latter has $S_{0}=0$, and hence even an arbitrary slow quench must lead to $\Delta E(\tau \rightarrow \infty) \neq 0$ and a $T \neq 0$ state, having a thermal entropy that at least accounts for the $T=0$ entropy of the initial NFL state. Hence, the observed power law, implying asymptotic approach to the adiabatic limit $\Delta E(\tau \rightarrow \infty)=0$ is surprising. It would suggest that $S_{0}$ is not manifested as thermal excitations in the final state for $\tau \rightarrow \infty$. Hence, we do not expect the power law to eventually persist for any finite $p$ for $\tau \rightarrow \infty$. We see an indication of only a weak deviation from the intermediate- $\tau$ power law for $p=10$ around $\tau \sim 30-40$ [Fig. 3(a), inset]. From the intermediate- $\tau$ power law, we can estimate a timescale, much longer than presently accessible in our calculations, where the scaling is expected to be violated due to $S_{0}$ (see Appendix D). This mechanism of the violation of adiabaticity due to $S_{0}$ in the large- $N$ limit is different from the hitherto known routes [26] of adiabaticity breaking. As we show in Appendix D, physics beyond the large- $N$ limit [43] suggests that the limits $\tau \rightarrow \infty$ and $N \rightarrow \infty$ do not commute, also indicating the absence of the adiabatic limit [26].

As shown in Fig. 3(c), a steady-state value of $E_{c \psi}(t)$, consistent with the thermal value is attained very rapidly within the NFL for any $\tau$. In contrast, a "glassy" behavior 
is seen within the FL, where $E_{c \psi}(t)$ relaxes very slowly for small $\tau$ but relaxes almost instantaneously for larger $\tau$ values [Fig. 3(d)].

\section{CONCLUSIONS}

In conclusion, our study of sudden and slow quenches in a large- $N$ model of NFL-FL transition reveal a remarkably rich nonequilibrium phase diagram and sharp contrasts between noninteracting FL and NFL phases. The sudden quench allows us to track the distinct evolutions of initially prepared well-defined quasipartile state in the NFL and FL phases and establish the existence of a dynamical phase transition which is different from the equilibrium NFL-FL quantum phase transition. In the context of slow quenches, unique features of the NFL-FL QPT and the low-temperature state of the SYK model, such as strongly interacting fermionic excitations and residual zero-temperature entropy, allow us to probe completely unexplored regime of out-of-equilibrium quantum many-body dynamics compared to previous studies of integrable and weakly integrable systems. These unusual features lead to remarkable intermediate nonanalytic scaling of excitation-energy production with the quench duration and the eventual breakdown of quantum adiabaticity. A natural future extension would be to go beyond large $N$ to study evolution for longer times $\sim N$.

\section{ACKNOWLEDGMENTS}

We thank Ehud Altman, Subroto Mukerjee, Diptiman Sen, Joel Moore, Vijay B. Shenoy, Emil Uzbashyan, Soumen Bag, Renato Dantas, and Paul A. McClarty for useful discussions. S.B. acknowledges support from The Infosys Foundation, India.

\section{APPENDIX A: NONEQUILIBRIUM GREEN'S FUNCTIONS AND KADANOFF-BAYM EQUATIONS}

\section{Interacting model}

We find the nonequilibrium Green's functions and the corresponding Kadanoff-Baym equations using the SchwingerKeldysh closed-contour formalism. To do this we write the Schwinger-Keldysh action [32] for the time-dependent Hamiltonian $\mathcal{H}$ in Eq. (1), i.e.,

$$
\begin{aligned}
\mathcal{S}= & \int_{\mathcal{C}} \mathrm{d} z\left[\sum_{i=1}^{N} \bar{c}_{i}(z)\left(\mathfrak{i} \partial_{z}+\mu\right) c_{i}(z)+\sum_{\alpha=1}^{M} \bar{\psi}_{\alpha}(z)\left(\mathfrak{i} \partial_{z}+\mu\right) \psi_{\alpha}(z)\right. \\
& -\frac{1}{(2 N)^{3 / 2}} \sum_{i j k l} J_{i j k l} \bar{c}_{i} \bar{c}_{j} c_{k} c_{l}-\frac{1}{M^{1 / 2}} \sum_{\alpha \beta} t_{\alpha \beta}^{\psi} \bar{\psi}_{\alpha} \psi_{\beta} \\
& \left.-\frac{f(z)}{(N M)^{1 / 4}} \sum_{i \alpha_{1}} V_{i \alpha} \bar{c}_{i} \psi_{\alpha}+V_{i \alpha}^{*} \bar{\psi}_{\alpha} c_{i}\right] .
\end{aligned}
$$

The contour variable $z$ lies on the usual Keldysh contour [32] (Fig. 4) with the forward (+) or backward ( - ) branches.

The explicit time dependence in the action is introduced via the function $f(z)=f(t)$. The nonequilibrium generating functional for obtaining time-dependent expectation values is defined as $\mathcal{Z}_{\text {neq }}=\int \mathcal{D}(c, \bar{c}, \bar{\psi}, \psi) e^{i \mathcal{S}[\bar{c}, c, \bar{\psi}, \psi]}$, under the

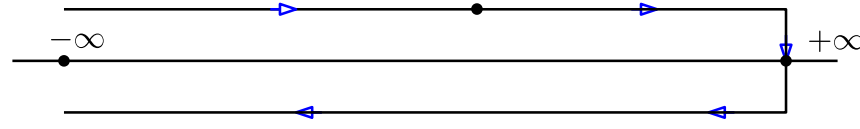

FIG. 4. Schwinger-Keldysh closed time contour: The closedtime Schwinger-Keldysh contour stretching from $-\infty$ to $+\infty$ forward in time and then backward from $+\infty$ to $-\infty$.

two usual assumptions. First, the initial density-matrix $\hat{\rho}$ is independent of any disorder, and all the disorder dependence has been pushed into the time-evolution operators. Second, the disorder is switched on sometime in the infinitely long past so that the system has enough time to equilibrate to the conditions created by the disorder-dependent Hamiltonian. These assumptions allow us to implement the averaging of $\mathcal{Z}_{\text {neq }}$ over all disorder realizations as follows:

$$
\begin{aligned}
\left\langle\mathcal{Z}_{\text {neq }}\right\rangle_{\text {dis }} & =\int \mathcal{D}(c, \bar{c}, \bar{\psi}, \psi)\left\langle e^{\mathrm{i} \mathcal{S}[\bar{c}, c, \bar{\psi}, \psi]}\right\rangle_{\mathrm{dis}} \\
& =\int \mathcal{D}(c, \bar{c}, \bar{\psi}, \psi) \int d[J, V, t] e^{\mathrm{i} \mathcal{S}} P[J] P[V] P[t]
\end{aligned}
$$

where $P[.] \mathrm{s}$ are the Gaussian probability distributions for the couplings $J_{i j k l}, t_{\alpha \beta}^{\psi}$, and $V_{i \alpha}$ appearing in Eq. (1). We perform the Gaussian integrals over the disorder distributions and define the large- $N$ fields,

$$
\begin{aligned}
G_{c}\left(z_{1}, z_{2}\right) & =-\frac{\mathfrak{i}}{N} \sum_{i} c_{i}\left(z_{1}\right) \bar{c}_{i}\left(z_{2}\right) \\
G_{\psi}\left(z_{1}, z_{2}\right) & =-\frac{\mathfrak{i}}{M} \sum_{\alpha} \psi_{\alpha}\left(z_{1}\right) \bar{\psi}_{\alpha}\left(z_{2}\right)
\end{aligned}
$$

that live on the contour and the corresponding Lagrange multipliers $\Sigma_{c, \psi}\left(z_{1}, z_{2}\right)$. Finally, after integrating out the fermions we end up with the action

$$
\begin{aligned}
\mathcal{S}[\Sigma, G]= & -\mathfrak{i} N \ln \operatorname{det}\left\{-\mathfrak{i}\left[\left(\mathfrak{i} \partial_{1}+\mu\right) \mathbf{1}-\boldsymbol{\Sigma}_{\mathbf{c}}\right]\right\} \\
& -\mathfrak{i} M \ln \operatorname{det}\left\{-\mathfrak{i}\left[\left(\mathfrak{i} \partial_{1}+\mu\right) \mathbf{1}-\boldsymbol{\Sigma}_{\psi}\right]\right\} \\
& \times \int_{\mathcal{C}} d z_{1} d z_{2}\left\{+\mathfrak{i} \frac{J^{2} N}{4} G_{c}\left(z_{2}, z_{1}\right)^{2} G_{c}\left(z_{1}, z_{2}\right)^{2}\right. \\
& +\mathfrak{i} \frac{t_{\psi}^{2} M f\left(z_{1}\right) f\left(z_{2}\right)}{2} G_{\psi}\left(z_{2}, z_{1}\right) G_{\psi}\left(z_{1}, z_{2}\right) \\
& +\mathfrak{i} V^{2} \sqrt{N M} f\left(z_{1}\right) f\left(z_{2}\right) G_{c}\left(z_{2}, z_{1}\right) G_{\psi}\left(z_{1}, z_{2}\right) \\
& +\Sigma_{c}\left(z_{1}, z_{2}\right)\left[-\mathfrak{i} N G_{c}\left(z_{2}, z_{1}\right)\right] \\
& \left.+\Sigma_{\psi}\left(z_{1}, z_{2}\right)\left[-\mathfrak{i} M G_{\psi}\left(z_{2}, z_{1}\right)\right]\right\},
\end{aligned}
$$

where the matrix $\left(\mathfrak{i} \partial_{1}+\mu\right) \mathbf{1}$ has elements of the form $\left(\mathfrak{i} \partial_{z_{1}}+\mu\right) \delta\left(z_{1}-z_{2}\right)$. The elements for matrices $\boldsymbol{\Sigma}_{\mathbf{c}}, \boldsymbol{\Sigma}_{\psi}$ are $\Sigma_{c}\left(z_{1}, z_{2}\right), \Sigma_{\psi}\left(z_{1}, z_{2}\right)$ respectively. The action $\mathcal{S}$ is extremized with respect to $G$ and $\Sigma$ to produce the large- $N$ saddle-point 
equations

$$
\begin{aligned}
\Sigma_{c}\left(z_{1}, z_{2}\right)= & J^{2} G_{c}\left(z_{1}, z_{2}\right)^{2} G_{c}\left(z_{2}, z_{1}\right) \\
& +\sqrt{p} V^{2} f\left(z_{1}\right) f\left(z_{2}\right) G_{\psi}\left(z_{1}, z_{2}\right) \\
\Sigma_{\psi}\left(z_{1}, z_{2}\right)= & t_{\psi}^{2} G_{\psi}\left(z_{1}, z_{2}\right)+\frac{V^{2}}{\sqrt{p}} f\left(z_{1}\right) f\left(z_{2}\right) G_{c}\left(z_{1}, z_{2}\right)
\end{aligned}
$$

and

$$
\left(\mathfrak{i} \partial_{z_{1}}+\mu\right) \delta\left(z_{1}-z_{2}\right)-\Sigma_{s}\left(z_{1}, z_{2}\right)=G_{s}^{-1}\left(z_{1}, z_{2}\right),
$$

where $G_{s}^{-1}$ is the inverse of the matrix $G_{s}$ with its elements given by $G_{s}\left(z_{1}, z_{2}\right)$ and $s=c, \psi$. We rewrite Eq. (A6) by multiplying with $G_{s}$ from the right and the left, respectively,

$$
\begin{aligned}
& \left(\mathfrak{i} \partial_{z_{1}}+\mu\right) G_{s}\left(z_{1}, z_{2}\right)-\int_{\mathcal{C}} d z \Sigma_{s}\left(z_{1}, z\right) G_{s}\left(z, z_{2}\right) \\
& \quad=\delta_{\mathcal{C}}\left(z_{1}, z_{2}\right), \\
& \left(-\mathfrak{i} \partial_{z_{2}}+\mu\right) G_{s}\left(z_{1}, z_{2}\right)-\int_{\mathcal{C}} d z G_{s}\left(z_{1}, z\right) \Sigma_{s}\left(z, z_{2}\right) \\
& \quad=\delta_{\mathcal{C}}\left(z_{1}, z_{2}\right),
\end{aligned}
$$

which are integrodifferential equations satisfied by $G_{s}\left(z_{1}, z_{2}\right)$ and where $\delta_{c}$ is the Dirac-delta function defined on the contour.

From the contour-ordered Green's function $G_{s}\left(z_{1}, z_{2}\right)$, we obtain the disorder-averaged real-time nonequilibrium Green's functions, greater $(>)$, lesser $(<)$, and retarded $R$, from $G_{s}\left(z_{1}, z_{2}\right)$ at the saddle point, e.g.,

$$
\begin{aligned}
G_{c}^{>}\left(t_{1}, t_{2}\right) & \equiv G_{c}\left(t_{1-}, t_{2+}\right)=-\mathfrak{i} \overline{\left\langle c_{i}\left(t_{1}\right) c_{i}^{\dagger}\left(t_{2}\right)\right\rangle}, \\
G_{c}^{<}\left(t_{1}, t_{2}\right) & \equiv G_{c}\left(t_{1+}, t_{2-}\right)=+\mathfrak{i} \overline{\left\langle c_{i}^{\dagger}\left(t_{2}\right) c_{i}\left(t_{1}\right)\right\rangle}, \\
G^{R}\left(t_{1}, t_{2}\right) & =-\mathfrak{i} \Theta\left(t_{1}-t_{2}\right) \overline{\left\langle\left\{c_{i}\left(t_{1}\right), c_{i}^{\dagger}\left(t_{2}\right)\right\}\right\rangle} \\
= & \Theta\left(t_{1}-t_{2}\right)\left[G^{>}\left(t_{1}, t_{2}\right)-G^{<}\left(t_{1}, t_{2}\right)\right], \\
G^{A}\left(t_{1}, t_{2}\right) & =+\mathfrak{i} \Theta\left(t_{2}-t_{1}\right) \overline{\left\langle\left\{c_{i}\left(t_{1}\right), c_{i}^{\dagger}\left(t_{2}\right)\right\}\right\rangle} \\
& =\Theta\left(t_{2}-t_{1}\right)\left[G^{<}\left(t_{1}, t_{2}\right)-G^{>}\left(t_{1}, t_{2}\right)\right] .
\end{aligned}
$$

and similarly for $G_{\psi}\left(z_{1}, z_{2}\right)$. The first (second) sign in the suffix of $G_{s}\left(t_{1 \pm}, t_{2 \mp}\right)$ indicates whether the $z_{1}\left(z_{2}\right)$ coordinate lies in the forward $(+\mathrm{ve})$ or backward $(-\mathrm{ve})$ branch of the contour.

a. Steady state. In a steady state the Green's functions are invariant under time translational, e.g., $G^{R}\left(t_{1}, t_{2}\right)=G^{R}\left(t_{1}-\right.$ $\left.t_{2}\right)=G^{R}(t)$.

b. Thermal equilibrium. For thermal equilibrium at a temperature $T$, in addition to the above steady-state condition, the Green's functions satisfy the FDT [32], e.g.,

$$
\mathfrak{i} G_{s}^{<}(\omega) /\left[2 \operatorname{Im} G_{s}^{R}(\omega)\right]=n_{F}(\omega, T),
$$

where $n_{F}(\omega, T)=1 /\left(e^{\omega / T}+1\right)$ is the Fermi function and $G_{\langle,\rangle, R, A}(\omega)$ are Fourier transforms of $G_{\langle,\rangle, R, A}(t)$ defined by

$$
G(\omega)=\int_{-\infty}^{\infty} G(t) e^{i \omega t} d \omega,
$$

The above conditions allow us to test whether a system, after undergoing a nonequilibrium process, has reached a steady state and whether the steady state is consistent with thermal equilibrium.

\section{The Kadanoff-Baym equations}

From Eq. (A7) and Eq. (A8), we obtain the time-evolution equations for $G_{s}^{>,<}$, e.g.,

$$
\begin{aligned}
\left(\mathfrak{i} \partial_{t_{1}}+\mu\right) G_{s}^{>}\left(t_{1}, t_{2}\right) & =\int_{\mathcal{C}} d z \Sigma_{s}\left(t_{1-}, z\right) G_{s}\left(z, t_{2+}\right) \\
& \equiv I_{s>}^{(1)}\left(t_{1}, t_{2}\right) \\
\left(-\mathfrak{i} \partial_{t_{2}}+\mu\right) G_{s}^{>}\left(t_{1}, t_{2}\right) & =\int_{\mathcal{C}} d z G_{s}\left(t_{1-}, z\right) \Sigma_{s}\left(z, t_{2+}\right) \\
& \equiv I_{s>}^{(2)}\left(t_{1}, t_{2}\right),
\end{aligned}
$$

where we have used the fact $\delta_{\mathcal{C}}\left(z_{1} \rightarrow t_{1-}, z_{2} \rightarrow t_{2+}\right)=0$. Finally, using Langreth rules [44], we get

$$
\begin{aligned}
I_{s>}^{(1)}\left(t_{1}, t_{2}\right)= & \int_{-\infty}^{t_{1}} \Sigma_{s}^{R}\left(t_{1}, t\right) G_{s}^{>}\left(t, t_{2}\right) d t \\
& +\int_{-\infty}^{t_{2}} \Sigma_{s}^{>}\left(t_{1}, t\right) G_{s}^{A}\left(t, t_{2}\right) d t \\
I_{s>}^{(2)}\left(t_{1}, t_{2}\right)= & \int_{-\infty}^{t_{1}} G_{s}^{R}\left(t_{1}, t\right) \Sigma^{>}\left(t, t_{2}\right) d t \\
& +\int_{-\infty}^{t_{2}} G_{s}^{>}\left(t_{1}, t\right) \Sigma_{s}^{A}\left(t, t_{2}\right) d t,
\end{aligned}
$$

where $\Sigma^{R}\left(\Sigma^{A}\right)$ is the retarded (advanced) self-energies, given by

$$
\begin{aligned}
& \Sigma^{R}\left(t_{1}, t_{2}\right)=\Theta\left(t_{1}-t_{2}\right)\left[\Sigma^{>}\left(t_{1}, t_{2}\right)-\Sigma^{<}\left(t_{1}, t_{2}\right)\right] \\
& \Sigma^{A}\left(t_{1}, t_{2}\right)=\Theta\left(t_{2}-t_{1}\right)\left[\Sigma^{<}\left(t_{1}, t_{2}\right)-\Sigma^{>}\left(t_{1}, t_{2}\right)\right],
\end{aligned}
$$

where, using Eq. (A5),

$$
\begin{aligned}
\Sigma_{c}^{>,<}\left(t_{1}, t_{2}\right)= & \Sigma_{c}\left(z_{1} \rightarrow t_{1-}, z_{2} \rightarrow t_{2+}\right) \\
= & J^{2} G_{c}^{>,<}\left(t_{1}, t_{2}\right)^{2} G_{c}^{<,>}\left(t_{2}, t_{1}\right) \\
& +\sqrt{p} V^{2} f\left(t_{1}\right) f\left(t_{2}\right) G_{\psi}^{>,<}\left(t_{1}, t_{2}\right) \Sigma_{\psi}^{>,<}\left(t_{1}, t_{2}\right) \\
= & \Sigma_{\psi}\left(z_{1} \rightarrow t_{1-}, z_{2} \rightarrow t_{2+}\right)=t_{\psi}^{2} G_{\psi}^{>,<}\left(t_{1}, t_{2}\right) \\
& +\frac{V^{2}}{\sqrt{p}} f\left(t_{1}\right) f\left(t_{2}\right) G_{c}^{>,<}\left(t_{1}, t_{2}\right) .
\end{aligned}
$$



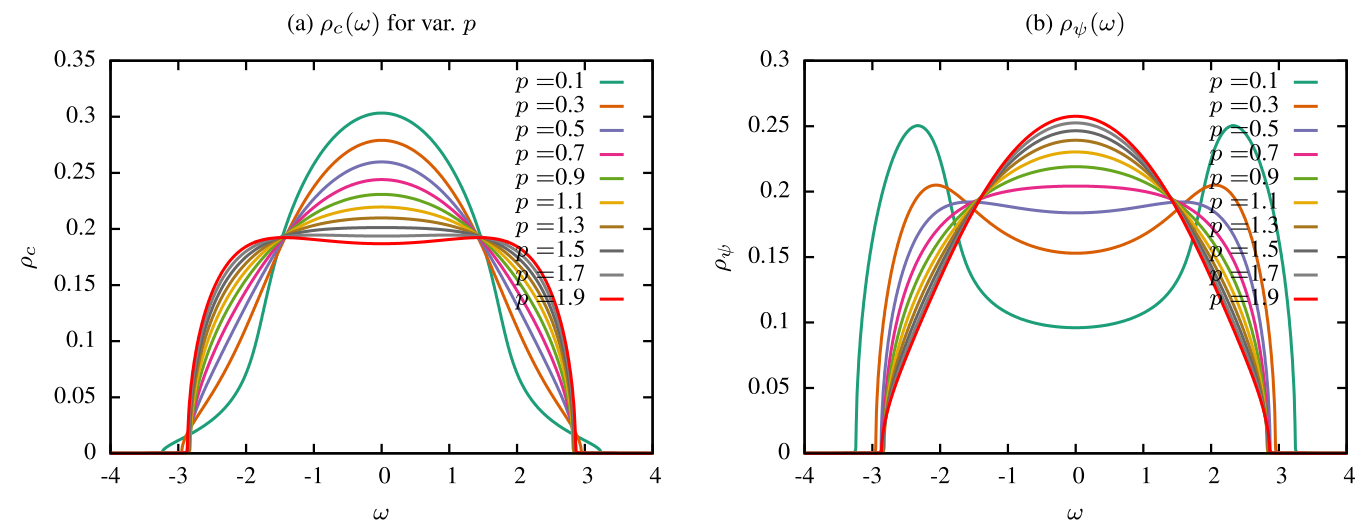

FIG. 5. Spectral functions for the noninteracting case at $T=0.04$. (a) The spectral functions for the $c$ fermions shown for multiple values of $p$. (b) Spectral function for the $\psi$ fermions, showing the soft-gap closing between $p=0.5$ and $p=0.7$.

Similarly, one can repeat the above analysis for the $G_{s}^{<}$ case. The integrodifferential equations for $G_{s}^{>}$and $G_{s}^{<}$can be stated together as

$$
\begin{aligned}
\left(\mathfrak{i} \partial_{t_{1}}+\mu\right) G_{s}^{>,<}\left(t_{1}, t_{2}\right)= & \int_{-\infty}^{t_{1}} \Sigma_{s}^{R}\left(t_{1}, t\right) G_{s}^{>,<}\left(t, t_{2}\right) d t \\
& +\int_{-\infty}^{t_{2}} \Sigma_{s}^{>,<}\left(t_{1}, t\right) G_{s}^{A}\left(t, t_{2}\right) d t \\
\left(-\mathfrak{i} \partial_{t_{2}}+\mu\right) G_{s}^{>,<}\left(t_{1}, t_{2}\right)= & \int_{-\infty}^{t_{1}} G_{s}^{R}\left(t_{1}, t\right) \Sigma_{s}^{>,<}\left(t, t_{2}\right) d t \\
& +\int_{-\infty}^{t_{2}} G_{s}^{>,<}\left(t_{1}, t\right) \Sigma_{s}^{A}\left(t, t_{2}\right) d t .
\end{aligned}
$$

This set of equations are called the KB equations, which, along with the relations given in Eq. (A17) and Eq. (A18), set up a closed system of equations that can be time evolved in the $t_{1}-t_{2}$ plane (see Fig. S1), starting from an initial condition for $G \mathrm{~s}$ and $\Sigma \mathrm{s}$.

\section{Noninteracting model}

Following procedure similar to that discussed above for the interacting model, we obtain the disorder-averaged Schwinger-Keldysh action for the noninteracting model of (2) and the saddle-point equations.

The large- $N$ self-energies are given by

$$
\begin{aligned}
\Sigma_{c}\left(z_{1}, z_{2}\right)= & t_{c}^{2} G_{c}\left(z_{1}, z_{2}\right) \\
& +\sqrt{p} V^{2} f\left(z_{1}\right) f\left(z_{2}\right) G_{\psi}\left(z_{1}, z_{2}\right) \\
\Sigma_{\psi}\left(z_{1}, z_{2}\right)= & t_{\psi}^{2} G_{\psi}\left(z_{1}, z_{2}\right) \\
& +\frac{V^{2}}{\sqrt{p}} f\left(z_{1}\right) f\left(z_{2}\right) G_{c}\left(z_{1}, z_{2}\right) .
\end{aligned}
$$

Consequently, a set of contour Kadanoff-Baym equations similar to the ones given in Eq. (A7) and Eq. (A8) is obtained. Using the Langreth rules the above equations involving contour indices $z_{1}, z_{2}$ can be changed to real-time variables $t_{1}, t_{2}$ to give the final Kadanoff-Baym equations [see Eq. (A19)] for the system with the following expressions for the self- energies:

$$
\begin{aligned}
& \Sigma_{c}^{>,<}\left(t_{1}, t_{2}\right)=t_{c}^{2} G_{c}^{>,<}\left(t_{1}, t_{2}\right)+\sqrt{p} V^{2} f\left(t_{1}\right) f\left(t_{2}\right) G_{\psi}^{>,<}\left(t_{1}, t_{2}\right) \\
& \Sigma_{\psi}^{>,<}\left(t_{1}, t_{2}\right)=t_{\psi}^{2} G_{\psi}^{>,<}\left(t_{1}, t_{2}\right)+\frac{V^{2}}{\sqrt{p}} f\left(t_{1}\right) f\left(t_{2}\right) G_{c}^{>,<}\left(t_{1}, t_{2}\right) .
\end{aligned}
$$

\section{APPENDIX B: GAP-CLOSING TRANSITIONS IN THE INTERACTING MODEL AND NONINTERACTING MODEL}

The spectral functions obtained from the equilibrium Green's functions (see the Supplemental Material [31], Sec. 2) for the connected system, i.e., $V=1$, for various $p$ values are discussed below. Figures 5(a) and 5(b) show the results for the noninteracting model, whereas Figs. 6(a) and 6(b) show the spectral functions for the interacting model. In the noninteracting case, we find that the spectral function for the $\psi$ fermions have a soft gap for smaller values of $p$, which closes completely around $p=0.5-0.7$. This is consistent with the dynamical transition that we observe for the sudden quench of the noninteracting model, which we discuss in the Supplemental Material [31], Sec. 4.3. The $c$-fermion spectral function does not have a soft gap for small values of $p$, but a soft gap begins to form near $p \sim 1.9$ as we increase $p$. This is expected, since the noninteracting model has an additional symmetry under $p \rightarrow 1 / p$ and $c \leftrightarrow \psi$. Moving on to the interacting model, we find a similar soft-gap-closing scenario taking place for the $\psi$-fermion spectral functions, as shown in Fig. 6(b). However, this time the gap closes completely around $p=1.6-2.6$, which is far from the equilibrium NFL-to-FL transition point of $p=1.0$. This is again consistent with the dynamical transition critical point [see Fig. 2(a) inset in the main text] that we find in the sudden quench of the interacting model. The $c$-fermion spectral functions have a highly peaked form around $\omega=0$, for smaller $p$ values, due to the presence of a divergent $T=0$ spectral function coming from the NFL fixed point. At higher values of $p$, the peak subsides and a gap begins to form in the spectral function. 
(a) $\rho_{c}(\omega)$ for var. $p$

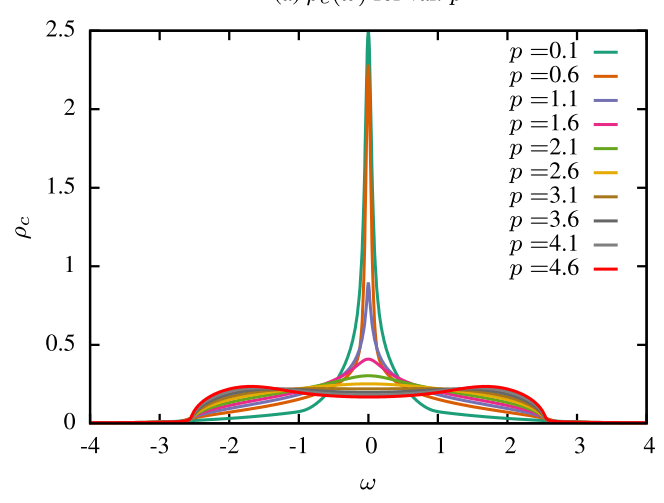

(b) $\rho_{\psi}(\omega)$

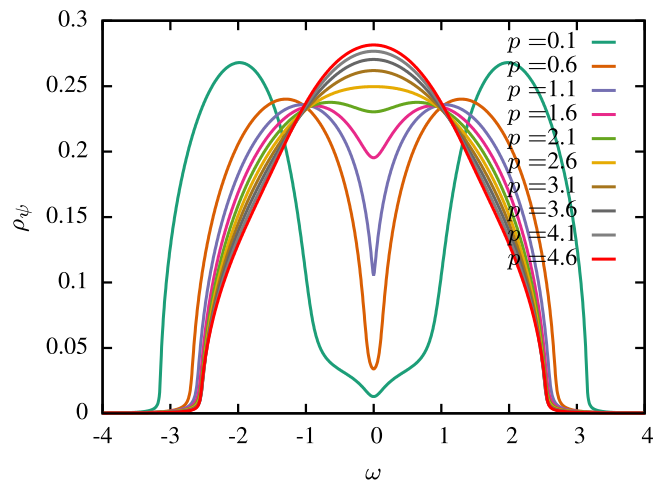

FIG. 6. Spectral functions for the interacting model at $T=0.04$. (a) Spectral function for the $c$ fermions for multiple values of $p$. (b) Spectral function for the $\psi$ fermions, showing the soft-gap closing somewhere between $p=1.6$ and $p=2.6$.

\section{APPENDIX C: EXCITATION ENERGY AS A FUNCTION OF QUENCH TIME FOR THE NONINTERACTING MODEL}

We also study the dependence of excitation energy $\Delta E$ on the quench duration $\tau$ for the slow quench of the noninteracting model of Eq. (2). We find that there exists a powerlaw relationship between $\Delta E$ and $\tau$ here as well, as shown in Fig. 7, which we report in the main text. However, the dependence of the power-law exponent $\eta$ on $p$ is qualitatively different from the interacting case as shown in Fig. 3(b) of the main text.

\section{APPENDIX D: BREAKDOWN OF ADIABATIC PERTURBATION THEORY AND THE ABSENCE OF ADIBATIC LIMIT}

Here we elaborate on the connection between the zerotemperature residual entropy of the SYK NFL and the absence

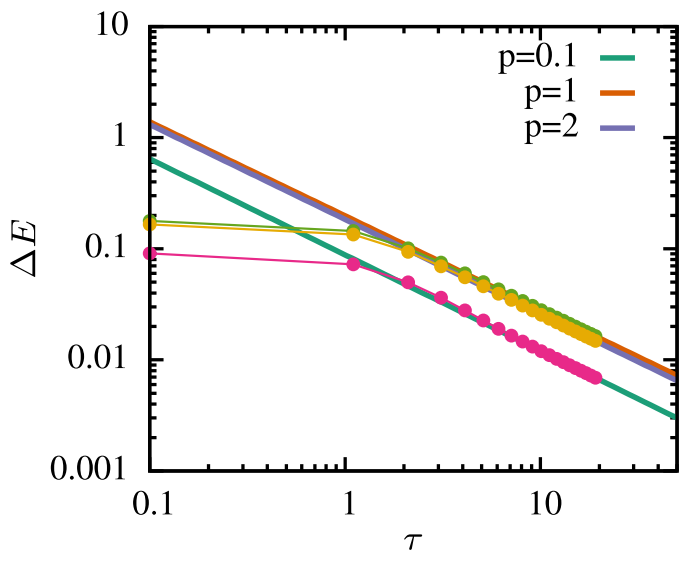

FIG. 7. Excitation energy vs. quench duration for the noninteracting model. The excitation energy $\Delta E$, produced in the slow quench, plotted as a function of quench duration $\tau$. The power-law dependence is clearly evident from the straight line fits to the log-log scale plot of $\Delta E$ vs. $\tau$. The slope of the straight lines are almost equal, making the exponent $\eta$ of the power law to have only a weak dependence on the site fraction $p$. This feature is in contrast with the $\eta-p$ dependence for the interacting model, where $\eta$ heavily depends on $p$ and has a minimum value at $p=1$, see Fig. 3(b). of the adiabatic limit for the slow quenches described in the main paper. In the large- $N$ limit, $S_{0}$ reflects the exponentially dense many-body energy spectrum near the ground state in the NFL phase [4], and the QPT in our model marks a transition from exponentially small many-body level spacing, $\Delta \sim e^{-S_{0}(p) N}$, in the NFL to $\Delta \sim 1 / N$ in the FL. Hence, the residual entropy $S_{0}$ cannot be thought of as a thermodynamic entropy strictly at $T=0$, i.e., when the $T \rightarrow 0$ limit is taken first, keeping $N$ finite and then $N \rightarrow \infty$ limit is taken, $S_{0}=$ 0 . In the large- $N$ description, the limit is taken the other way around, and it captures the exponentially dense manybody level spectrum near the ground state in the NFL phase. However, at any nonzero temperature $\left(\gtrsim e^{-S_{0} N}\right)$, which could be infinitesimally small in the large- $N$ limit, $S_{0}$ is the true thermodynamic entropy. Hence, we expect this entropy to be manifested in the large- $N$ nonequilibrium dynamics during a slow quench, implying the absence of the adiabatic limit. However, as mentioned in the main text, surprisingly we find the intermediate- $\tau$ nonanalytic power-law scaling, which seems to mask the effect of residual entropy. In the following, we first try to estimate the power law from the so-called adiabatic perturbation theory [25,38-40], which has been previously used for noninteracting and weakly interacting systems. We show that the adibatic perturbation theory cannot explain the $p$-dependent exponent directly obtained from the direct nonequilibrium evolution, discussed in the main text. Furthermore, we discuss the possible modes of violation of the adiabaticity in the large- $N$ limit, as well as beyond it.

\section{Adiabatic perturbation theory in the large- $N$ limit}

In the adiabatic perturbation theory [25,38-40], the timedependent term [Eq. (1c)] is treated as a perturbation assuming a weak strength of the ramp in Eq. (1), i.e., $f(t)=$ $\Delta f r(t / \tau)$ with $\Delta f \ll 1$. To this end, we obtain the energy generated during quench [38] as

$$
\Delta E(\tau) \simeq \Delta f^{2} \int_{0}^{\infty} \frac{d \omega}{\omega} \mathcal{A}_{\mathcal{V}}(\omega) R(\omega \tau)+\mathcal{O}\left(\Delta f^{2}\right) .
$$

Here $R(x)=\left|\int_{0}^{1} d s r^{\prime}(s) e^{i x s}\right|^{2}$ and $r^{\prime}(x)$ is derivative of the ramp function. $\mathcal{A}_{\mathcal{V}}(\omega)$ is a $T=0$ spectral function corresponding to the disordered-averaged imaginary-time correlation function of the operator $\mathcal{V}=(N M)^{-1 / 4} \sum_{i \alpha}\left(V_{i \alpha} c_{i}^{\dagger} \psi_{\alpha}+\right.$ 
h.c. $)$, i.e., $-\overline{\left\langle\mathcal{T}_{\tau} \mathcal{V}(\tau) \mathcal{V}(0)\right\rangle}$. This can be computed in the large$N$ limit, and we obtain

$$
\mathcal{A}_{\mathcal{V}}(\omega)=\frac{2 \sqrt{p}}{1+p} V^{2} \int_{0}^{\omega} d \omega^{\prime} \rho_{c}\left(\omega^{\prime}\right) \rho_{\psi}\left(\omega^{\prime}-\omega\right),
$$

where $\rho_{c}(\omega)$ and $\rho_{\psi}(\omega)$ are the spectral functions of the SYK and lead fermions, respectively, for the uncoupled systems before the quench. For long quench time $\tau \gg \Omega^{-1}$, where $\Omega \approx J, t_{\psi}$, we can use the low-energy forms, $\rho_{c}(\omega) \sim|\omega|^{-1 / 2}$ and $\rho_{\psi}(\omega) \sim$ const, for $\omega \ll \Omega$. These give $\mathcal{A}_{\mathcal{V}}(\omega) \sim \omega^{1 / 2}$. It can be shown [38] that $R(x \rightarrow \infty) \sim x^{-2 n}$, where $n \geqslant 1$ is the order of the derivative discontinuity in the ramp (see the Supplemental Material [31], Sec. 5.1). As a result,

$$
\Delta E(\tau) \sim \tau^{-1 / 2} \int_{0}^{\Omega \tau} d x x^{-1 / 2} R(x) .
$$

Moreover, the integral above is convergent for $\tau \rightarrow \infty$ since $2 n>1 / 2$. Hence, the adiabatic perturbation theory predicts a nonanalytic power law with exponent $\eta=1 / 2$ for any $p$. This, of course, does not agree with the strongly nonmonotonic $\eta(p)$ obtained from the direct nonequilibrium calculations [Fig. 3(b)]. Hence, the adiabatic perturbation theory does not work in our case. The theory assumes a nondegenerate ground state [38], and it is an interesting question whether such a theory could at all be applied to a phase with exponentially dense many-body spectrum near the ground state, even though some of the effect of the dense spectrum is incorporated in the large- $N$ single-particle density of states.

\section{Breakdown of adiabaticity in the large- $N$ limit}

As discussed in the main text, the presence of a residual entropy in the SYK fermions, prior to the quench, implies that a finite amount of excitation energy $\Delta E>0$ must be produced even in the $\tau \rightarrow \infty$ limit. Therefore, the power-law relationship $\Delta E \sim \tau^{-\eta}$, in principle, should break down at some large $\tau$. We now provide a route to find an estimate for the time $\tau_{\text {break }}$ at which we can expect the power-law behavior to break down. This can be done by assuming an isoentropic (the initial and final entropies are taken to be equal) limit of the quench process. We first calculate the temperature $T_{f}$ necessary for the final Hamiltonian $H_{f}$ to hold the initial entropy $S_{i}$ by solving the equilibrium problem and demanding

$$
S_{i}=S_{f}\left(T_{f}\right) .
$$

Using the definition of $\Delta E$ given in the main text, we can estimate $\tau_{\text {break }}$ from the condition $\Delta E\left(\tau_{\text {break }}\right) \approx \Delta E_{T_{f}}=$ $\left\langle\mathcal{H}\left(\tau_{\text {break }}\right)\right\rangle_{T_{f}}-\left\langle\mathcal{H}\left(\tau_{\text {break }}\right)\right\rangle_{T_{i}}$. The latter is the excitation energy produced by converting the initial entropy to thermal excitations, and the excitations generated by the quench cannot be lower than $\Delta E_{T_{f}}$. This leads to

$$
\tau_{\text {break }}(p)=\left[\frac{\operatorname{Tr}\left[\hat{\rho}\left(T_{f}\right) H_{f}\right]-\operatorname{Tr}\left[\hat{\rho}\left(T_{i}\right) H_{f}\right]}{\alpha}\right]^{-1 / \eta},
$$

where $\alpha$ can be extracted from power-law fits to $\Delta E$ using

$$
\Delta E=\alpha \tau^{-\eta} .
$$

Performing such an estimate for the $p=1.5$ case, we find $\tau_{\text {break }} \sim 200$, a time which is not easily accessible using the numerical algorithm in the Supplemental Material [31], Sec. 1 .

\section{The adiabatic perturbation theory beyond large $N$ and the absence of adiabatic limit}

Within the adiabatic perturbation theory discussed above, we can go beyond the large- $N$ theory by incorporating some finite- $N$ corrections, at least due to the single-particle level spacing, following Ref. [43]. It is still not known how to incorporate the effects of many-body level spacing. Nevertheless, it has been shown in Ref. [43] that the SYK spectral function $\rho_{c}(\omega)$ changes from the divergent $|\omega|^{-1 / 2}$ behavior to $\rho_{c}(\omega) \sim \Delta_{s}^{-1}|\omega|^{1 / 2}$ for $\omega \ll \Delta_{s} \sim J /(N \ln N)$. The large prefactor $N \ln N$ in the $\sqrt{|\omega|}$ dependence presumably arises from the dense spectrum, even though the density of states is suppressed at low energies. We obtain $\mathcal{A}_{\mathcal{V}}(\omega) \sim \omega^{3 / 2}$ for $\omega \ll \Delta_{s}$, giving

$$
\begin{gathered}
\Delta E(\tau) \sim \tau^{-3 / 2} N \ln N \int_{0}^{\Delta_{s} \tau} d x x^{1 / 2} R(x) \\
+\tau^{-1 / 2} \int_{\Delta_{s} \tau}^{\Omega \tau} d x x^{-1 / 2} R(x) .
\end{gathered}
$$

Hence, keeping $N$ fixed, we obtain $\Delta E(\tau) \sim \tau^{-3 / 2} N \ln N$ for $\tau \rightarrow \infty$. Clearly, the limits $\tau \rightarrow \infty$ and $N \rightarrow \infty$ do not commute. This indicates that the adiabatic limit cannot be reached.
[1] S. Sachdev, Quantum Phase Transitions, 2nd ed. (Cambridge University Press, Cambridge, UK, 2011).

[2] S. Sachdev and J. Ye, Gapless Spin-Fluid Ground State in a Random Quantum Heisenberg Magnet, Phys. Rev. Lett. 70, 3339 (1993).

[3] A. Kitaev, A simple model of quantum holography, Talks at KITP, April 7, 2015 and May 27, 2015, http://online.kitp.ucsb. edu/online/entangled15/kitaev/.

[4] J. Maldacena and D. Stanford, Remarks on the Sachdev-YeKitaev model, Phys. Rev. D 94, 106002 (2016).

[5] Y. Gu, A. Lucas, and Xiao-Liang Qi, Spread of entanglement in a Sachdev-Ye-Kitaev chain, J. High Energ. Phys. 09 (2017) 120.
[6] S. Banerjee and E. Altman, Solvable model for a dynamical quantum phase transition from fast to slow scrambling, Phys. Rev. B 95, 134302 (2017).

[7] S.-K. Jian and H. Yao, Solvable Sachdev-Ye-Kitaev Models in Higher Dimensions: From Diffusion to Many-Body Localization, Phys. Rev. Lett. 119, 206602 (2017).

[8] X.-Y. Song, C.-M. Jian, and L. Balents, Strongly Correlated Metal Built from Sachdev-Ye-Kitaev Models, Phys. Rev. Lett. 119, 216601 (2017).

[9] R. A. Davison, W. Fu, A. Georges, Y. Gu, K. Jensen, and S. Sachdev, Thermoelectric transport in disordered metals without quasiparticles: The Sachdev-Ye-Kitaev models and holography, Phys. Rev. B 95, 155131 (2017). 
[10] A. A. Patel, J. McGreevy, D. P. Arovas, and S. Sachdev, Magnetotransport in a Model of a Disordered Strange Metal, Phys. Rev. X 8, 021049 (2018).

[11] D. Chowdhury, Y. Werman, E. Berg, and T. Senthil, Translationally Invariant Non-Fermi-Liquid Metals with Critical Fermi Surfaces: Solvable Models, Phys. Rev. X 8, 031024 (2018).

[12] A. Haldar, S. Banerjee, and V. B. Shenoy, Higher-dimensional Sachdev-Ye-Kitaev non-Fermi liquids at Lifshitz transitions, Phys. Rev. B 97, 241106(R) (2018).

[13] A. Haldar and V. B. Shenoy, Strange half-metals and Mott insulators in Sachdev-Ye-Kitaev models, Phys. Rev. B 98, 165135 (2018).

[14] S. Sachdev, Holographic Metals and the Fractionalized Fermi Liquid, Phys. Rev. Lett. 105, 151602 (2010).

[15] S. Sachdev, Bekenstein-Hawking Entropy and Strange Metals, Phys. Rev. X 5, 041025 (2015).

[16] J. Maldacena, S. H. Shenker, and D. Stanford, A bound on chaos, J. High Energy Phys. 08 (2016) 106.

[17] A. Kitaev and S. J. Suh, Statistical mechanics of a twodimensional black hole, J. High Energ. Phys. 05 (2019) 198

[18] M. Eckstein, M. Kollar, and P. Werner, Thermalization After an Interaction Quench in the Hubbard Model, Phys. Rev. Lett. 103, 056403 (2009).

[19] O. Can, E. M. Nica, and M. Franz, Charge transport in graphene-based mesoscopic realizations of Sachdev-Ye-Kitaev models, Phys. Rev. B 99, 045419 (2019).

[20] O. Parcollet and A. Georges, Transition from Overscreening to Underscreening in the Multichannel Kondo Model: Exact Solution at Large N, Phys. Rev. Lett. 79, 4665 (1997).

[21] F. H. L. Essler and M. Fagotti, Quench dynamics and relaxation in isolated integrable quantum spin chains, J. Stat. Mech. (2016) 064002.

[22] L. D'Alessio, Y. Kafri, A. Polkovnikov, and M. Rigol, From quantum chaos and eigenstate thermalization to statistical mechanics and thermodynamics, Adv. Phys. 65, 239 (2016).

[23] M. Moeckel and S. Kehrein, Interaction Quench in the Hubbard Model, Phys. Rev. Lett. 100, 175702 (2008).

[24] M. Moeckel and S. Kehrein, Real-time evolution for weak interaction quenches in quantum systems, Ann. Phys. 324, 2146 (2009).

[25] J. Dziarmaga, Dynamics of a quantum phase transition and relaxation to a steady state, Adv. Phys. 59, 1063 (2010).

[26] A. Polkovnikov, K. Sengupta, A. Silva, and M. Vengalattore, Colloquium: Nonequilibrium dynamics of closed interacting quantum systems, Rev. Mod. Phys. 83, 863 (2011).

[27] A. Eberlein, V. Kasper, S. Sachdev, and J. Steinberg, Quantum quench of the Sachdev-Ye-Kitaev model, Phys. Rev. B 96, 205123 (2017).
[28] J. Sonner and M. Vielma, Eigenstate thermalization in the Sachdev-Ye-Kitaev model, J. High Energy Phys. 11 (2017) 149.

[29] I. Kourkoulou and J. Maldacena, Pure states in the SYK model and nearly- $A d S_{2}$ gravity, (2017) arXiv:1707.02325 [hep-th].

[30] R. Bhattacharya, D. P. Jatkar, and N. Sorokhaibam, Quantum quenches and thermalization in SYK models, J. High Energ. Phys. 07 (2019) 066.

[31] See Supplemental Material at http://link.aps.org/supplemental/ 10.1103/PhysRevResearch.2.013307 for additional information.

[32] A. Kamenev, Field Theory of Non-Equilibrium Systems, 1st ed. (Cambridge University Press, Cambridge, UK, 2011).

[33] G. Stefanucci and R. van Leeuwen, Nonequilibrium Many-Bod Theory of Quantum Systems: A Modern Introduction, 1st ed. (Cambridge University Press, Cambridge, UK, 2013).

[34] We keep the initial temperature of the SYK subsystem low but finite. Because of the divergent spectral density at $T=0$, the Green's function for SYK fermions in the initial equilibrium state cannot be obtained numerically at strictly zero temperature.

[35] M. Stark and M. Kollar, Kinetic description of thermalization dynamics in weakly interacting quantum systems, (2013) arXiv:1308.1610 [cond-mat.str-el].

[36] S. R. Manmana, S. Wessel, R. M. Noack, and A. Muramatsu, Strongly Correlated Fermions After a Quantum Quench, Phys. Rev. Lett. 98, 210405 (2007).

[37] J. Polchinski and V. Rosenhaus, The spectrum in the SachdevYe-Kitaev model, J. High Energy Phys. 04 (2016) 001.

[38] M. Eckstein and M. Kollar, Near-adiabatic parameter changes in correlated systems: influence of the ramp protocol on the excitation energy, New J. Phys. 12, 055012 (2010).

[39] A. Polkovnikov, Universal adiabatic dynamics in the vicinity of a quantum critical point, Phys. Rev. B 72, 161201(R) (2005).

[40] C. De Grandi, V. Gritsev, and A. Polkovnikov, Quench dynamics near a quantum critical point, Phys. Rev. B 81, 012303 (2010).

[41] A. Almheiri, A. Milekhin, and B. Swingle, Universal constraints on energy flow and SYK thermalization, (2019) arXiv:1912.04912 [hep-th].

[42] S. Banerjee, A. Haldar, and S. Bera (unpublished).

[43] D. Bagrets, A. Altland, and A. Kamenev, Sachdev-Ye-Kitaev model as Liouville quantum mechanics, Nucl. Phys. B 911, 191 (2016).

[44] D. C. Langreth and J. W. Wilkins, Theory of spin resonance in dilute magnetic alloys, Phys. Rev. B 6, 3189 (1972). 